\title{
A Collaborative European Approach to Accelerating Translational Marine Science
}

\author{
Philip Brennecke ${ }^{1}$, Maria I. Ferrante 2 (1D) Ian A. Johnston ${ }^{3}$ and David Smith ${ }^{4, *(1)}$ \\ 1 Leibniz-Research Institute for Molecular Pharmacology, Robert-Roessle-Strasse 10, 13125 Berlin, Germany; \\ pbrennecke@fmp-berlin.de \\ 2 Integrative Marine Ecology, Stazione Zoologica Anton Dohrn, Villa Comunale, 80121 Napoli, Italy; \\ mariella.ferrante@szn.it \\ 3 Scottish Oceans Institute, University of St Andrews, St Andrews, Fife, Scotland KY16 8LB, UK; \\ iaj@st-andrews.ac.uk \\ 4 CAB International, Bakeham Lane, Egham, Surrey TW20 9TY, UK \\ * Correspondence: d.smith@cabi.org
}

Received: 31 May 2018; Accepted: 2 July 2018; Published: 5 July 2018

\begin{abstract}
Marine environments account for over $90 \%$ of the biosphere and hold tremendous potential for biotechnological applications and drug discovery. To fully exploit this potential and develop interesting discoveries into useful molecular tools and successful products, a multidisciplinary approach is indispensable. Here, we introduce the European Marine Biological Research Infrastructure Cluster (EMBRIC), a novel collaborative initiative that aims to facilitate translational marine science and remove existing bottlenecks that are currently impeding blue innovation. In the context of this initiative, pilot projects have been designed to test the functionality of the cluster focusing on two specific sectors of marine biotechnology: (i) the discovery and exploitation of marine natural products and (ii) the marker-assisted selection of desirable traits in aquaculture. EMBRIC brings together the expertise of six European Research Infrastructures on accessing the potential of marine organisms, specifically on the $99 \%$ of bacteria yet to be grown in culture, the microalgae, finfish, and shellfish. It improves the throughput and efficiency of workflows for discovery of novel marine products and facilitates projects that require an interdisciplinary approach. The objective is to develop coherent chains of high quality services for access to biological, analytical, and data resources by deploying common underpinning technologies and practices. The connection of academic science with industry is being strengthened by engaging companies, as well as geographically separated public and private-sector communities in the domain of marine biotechnology, and by federating technology transfer services amongst the players involved.
\end{abstract}

Keywords: marine biotechnology; microorganisms; microalgae; finfish; research infrastructure; discovery pipeline; bioactive compounds

\section{Introduction}

In addition to providing food for mankind and livestock, the oceans represent a vast reservoir of genetic diversity that can potentially be harnessed to drive scientific innovation and develop new products. The establishment of numerous marine stations since the 19th century has greatly facilitated access to marine organisms; however, most stations are relatively remote from major centres of life sciences research, which provide the interdisciplinary skill sets and large scale infrastructure required for modern biological research. This problem has become even more acute following the advent of 'omics' technologies. European Research Infrastructures represent an attractive means of bridging this gap by providing leading expertise and state-of-the-art life sciences facilities to marine biologists, and by making marine model organisms accessible to a wide range of researchers. 


\section{European Research Infrastructures: Promoting Large Scale and Interdisciplinary Research}

Since its inauguration in 2002, the European Strategy Forum on Research Infrastructures (ESFRI) has been driving the development of numerous Research Infrastructures with the aim of promoting multidisciplinary collaborations across Europe in order to accelerate scientific discoveries. The underlying idea is very simple: a Research Infrastructure typically combines expertise and capital-intensive instrumentation used in a certain field of research and makes those resources available to the broad scientific community, which in turn allows non-specialist users to readily tap into new research areas without having to start from scratch every time they want to supplement their projects with an experiment outside their core competencies or capacities. This powerful approach, which is somewhat reminiscent of smaller-scale core facilities belonging to individual universities or research institutes, has been deemed widely useful by the scientific community, and the latest ESFRI Roadmap published in 2016 now comprises 21 Research Infrastructures that cover a broad scientific spectrum, including, amongst others, the fields of environmental, health, and food research (see https:/ / ec.europa.eu/research/infrastructures/index_en.cfm?pg=esfri).

\section{EMBRIC: Driving Innovation through Collaboration}

This research infrastructure approach is particularly attractive for specialized research centres, such as marine stations, that, although highly skilled in their field of expertise, may lack the know-how to perform the downstream experiments that are indispensable for translating basic research findings into molecular tools and marketable products. The Horizon 2020 EU-funded European Marine Biological Research Infrastructure Cluster (EMBRIC) emerged in 2015 in order to close this gap and boost translational research. The EMBRIC cluster aims to build a dynamic network of partners from established and emerging research infrastructures to advance the field of marine biotechnology. The EMBRIC project includes experimental pilot projects focusing on two specific sectors of marine biotechnology: (i) aquaculture genetics and breeding, and (ii) discovery and exploitation of marine natural products from microalgae and marine microbes (Figure 1). A total of 29 scientific partners (i.e., universities, research institutes, non-profit organizations, and SMEs), most of which are also members of a European Research Infrastructure, have joined forces and generated a comprehensive map of all resources available within the EMBRIC network with the ultimate goal to make those resources (i.e., facilities, technologies, marine organisms, data, and experimental know-how) broadly accessible to the scientific community (see http:/ /www.embric.eu/).

In a nutshell, EMBRIC's mission is to provide continuous support throughout each and every step of the marine discovery workflow-from the discovery of novel marine organisms all the way to the final molecular tool or commercial product. This is possible because most of the partner institutions are already embedded in one of the six participating Research Infrastructures that collectively cover the relevant scientific disciplines for marine biotechnology (see Table 1). This combination of expertise has allowed EMBRIC to reach the critical mass necessary to effectively provide support in a variety of interconnecting areas-ranging from characterization and cataloguing of novel marine organisms through to the extraction of promising biomolecules, optimization experiments, and technology transfer support. EMBRIC's mission is not restricted to marine biologists. Translating any basic research result into a useful tool generally requires a cross-disciplinary approach and typically involves multiple partners from different research fields. Hence, EMBRIC engages extensively in showcasing its marine research results to interested stakeholders within and outside the immediate network such as, for example, pharmaceutical and biotech companies. By establishing such contacts between people who would otherwise not meet, EMBRIC envisages enabling numerous mutually beneficial collaborations throughout its lifetime that will benefit both the peripheral maritime region and the application-oriented collaborator usually located more inland. In this context, it is EMBRIC's broad positioning across different scientific disciplines that will allow for an effective selection of suitable collaborators and subsequent matchmaking. 

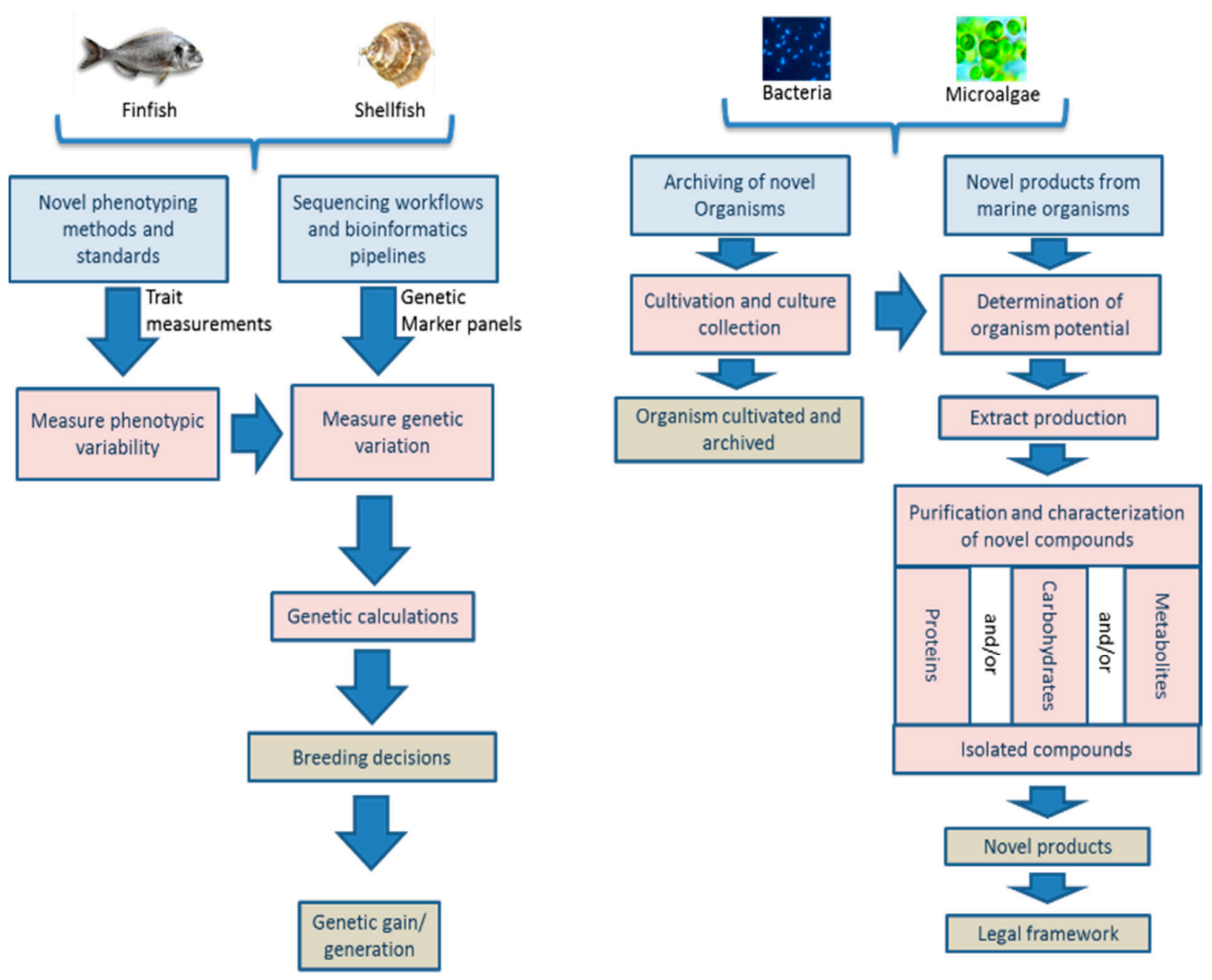

Figure 1. The EMBRIC pilot discovery pipelines in aquaculture genetics and breeding, and discovery and exploitation of marine natural products from microalgae and marine microbes.

Table 1. European Research Infrastructures participating in EMBRIC and their respective fields of expertise.

\begin{tabular}{cl}
\hline Research Infrastructure & \multicolumn{1}{c}{ Field of Expertise } \\
\hline EMBRC ERIC & Isolation, characterization, cultivation, and cataloguing of marine organisms \\
\hline MIRRI & Isolation, characterization, cultivation, and cataloguing of marine microorganisms \\
\hline EU-OPENSCREEN ERIC & $\begin{array}{l}\text { Bioprofiling, high throughput screening, and medicinal chemistry approaches for } \\
\text { hit-to-lead optimization }\end{array}$ \\
\hline Elixir & Data handling and long-term storage of data in databases \\
\hline AQUAEXCEL 2020 & Integration and standardisation of tools for aquaculture research \\
\hline RISIS & $\begin{array}{l}\text { Science and innovation studies, investigation of research and innovation } \\
\text { dynamics, and policies }\end{array}$ \\
\hline
\end{tabular}

\section{Introducing the Players Involved}

Usually, blue innovation starts in the ocean with the identification of novel organisms with interesting characteristics. To approach this critical first step in a systematic manner, the European Marine Biological Resource Centre (EMBRC, see http://www.embrc.eu/) Research Infrastructure and the Microbial Resources Research Infrastructure (MIRRI, see http: / www.mirri.org) are jointly focusing their efforts on isolating, characterizing, and cataloguing novel marine species. To this end, both partners provide access to marine and coastal ecosystems, marine model organisms, and 
numerous culture collections. MIRRI has a clear focus on microorganisms, while EMBRC covers a broader spectrum of marine species. Hence, these two research infrastructures collectively ensure maximum coverage of marine biodiversity within the EMBRIC network.

A major constraint in accessing the potential of marine microorganisms is our inability to isolate and subsequently grow them. This aspect is being addressed by MIRRI, and novel tools to access these organisms and their chemistry are being explored. Access to state of the art equipment such as novel 'omics' and imaging technologies allows EMBRC and MIRRI to perform thorough characterization of newly isolated species, and longstanding expertise in cultivation and cataloguing of marine organisms ensures that interesting biomaterial is readily accessible and available in appropriate quantities for downstream experiments.

Once a novel organism has been characterized, catalogued, and cultivated successfully, EU-OPENSCREEN, the European Research Infrastructure for high throughput screening and medicinal chemistry (see http:/ / www.eu-openscreen.eu/), provides support for scientists who are interested in isolating and testing marine natural products for interesting bioactivities. Here, the expertise of EU-OPENSCREEN includes assistance during extraction and fractionation of natural products, as well as initial bioprofiling in order to obtain bioactivity profiles from newly isolated marine biomolecules. Moreover, EU-OPENSCREEN invites scientists to subsequently donate their newly discovered compounds to the EU-OPENSCREEN compound library. Once archived in this library, the donated compounds will be used routinely in all subsequent high throughput screens performed at a total of 20 European EU-OPENSCREEN partner sites. The donating scientist automatically receives updates every time a donated compound generates a hit in a screening campaign. In this way, the compound-providing scientist gets a better chemical understanding of the donated compound and its bioactivity profile over time. Moreover-subsequent to a primary hit in a biological assay-EU-OPENSCREEN also offers support in hit-to-lead optimization, which allows for the optimization of desired compound properties and thus allows for the transformation of a promising candidate compound into a useful molecular tool or drug candidate.

As a lot of scientific results are produced on the route to discovery, managing and safeguarding data along the way is of utmost importance to ensure reproducibility and successful translation of basic research results. The Research Infrastructure ELIXIR (see https: / www.elixir-europe.org/) is specialized in handling large amounts of biological data and ensures effective collection, quality control, and secure storage of, as well as ready access to, all data generated within the EMBRIC network. In this context, it is a key challenge to make as much data available for public use as possible while, at the same time, restricting access to those datasets that will be used to generate intellectual property.

Two additional infrastructures bring added depth to the EMBRIC consortium: AQUAEXCEL2020 (see http:/ / www.aquaexcel2020.eu/) provides a pan-European association of aquaculture research facilities, whilst the Research Infrastructure for Research and Innovation Policy Studies (RISIS, see http://risis.eu/) addresses research and innovation dynamics. In the context of EMBRIC, AQUAEXCEL2020, a European Commission Integrated Infrastructure Initiatives project that focuses on marker-assisted genetic selection in aquaculture of finfish, provides the EMBRIC research community with biological resources (finfish) and access to a wide range of production systems (including recirculation, flow-through, hatchery, cage, and pond systems), as well as associated expertise.

The Integrating Activity project RISIS is specialized in the analysis of innovation ecosystems across Europe and, in close collaboration with the Technology Transfer Officers of EMBRIC partner institutions, is involved in establishing the technology transfer identity of EMBRIC. Generation of intellectual property (IP) is a complex task, and experimental scientists usually get exposed to IP-related issues only to a limited extent. Hence, in order to be able to support scientists at advanced stages of their marine discovery projects, EMBRIC combines expertise and best practices regarding intellectual property. To this end, Technology Transfer Officers of the 29 EMBRIC partner institutions meet on a regular basis to nurture exchange of expertise and establish common guidelines for generation of intellectual property. These guidelines, in conjunction with EMBRIC's large network of Technology 
Transfer Officers, will ensure a quick turnaround time for all issues related to technology transfer and generation of intellectual property. In addition, RISIS is also in the process of gathering, consolidating, harmonizing, and making available a total of 14 databases, covering topics such as the dynamics of innovation and the evaluation of research policies (http:/ / datasets.risis.eu/).

Figure 2 summarizes the engagement of the participating Research Infrastructures and industrial partners in the EMBRIC discovery pipelines.

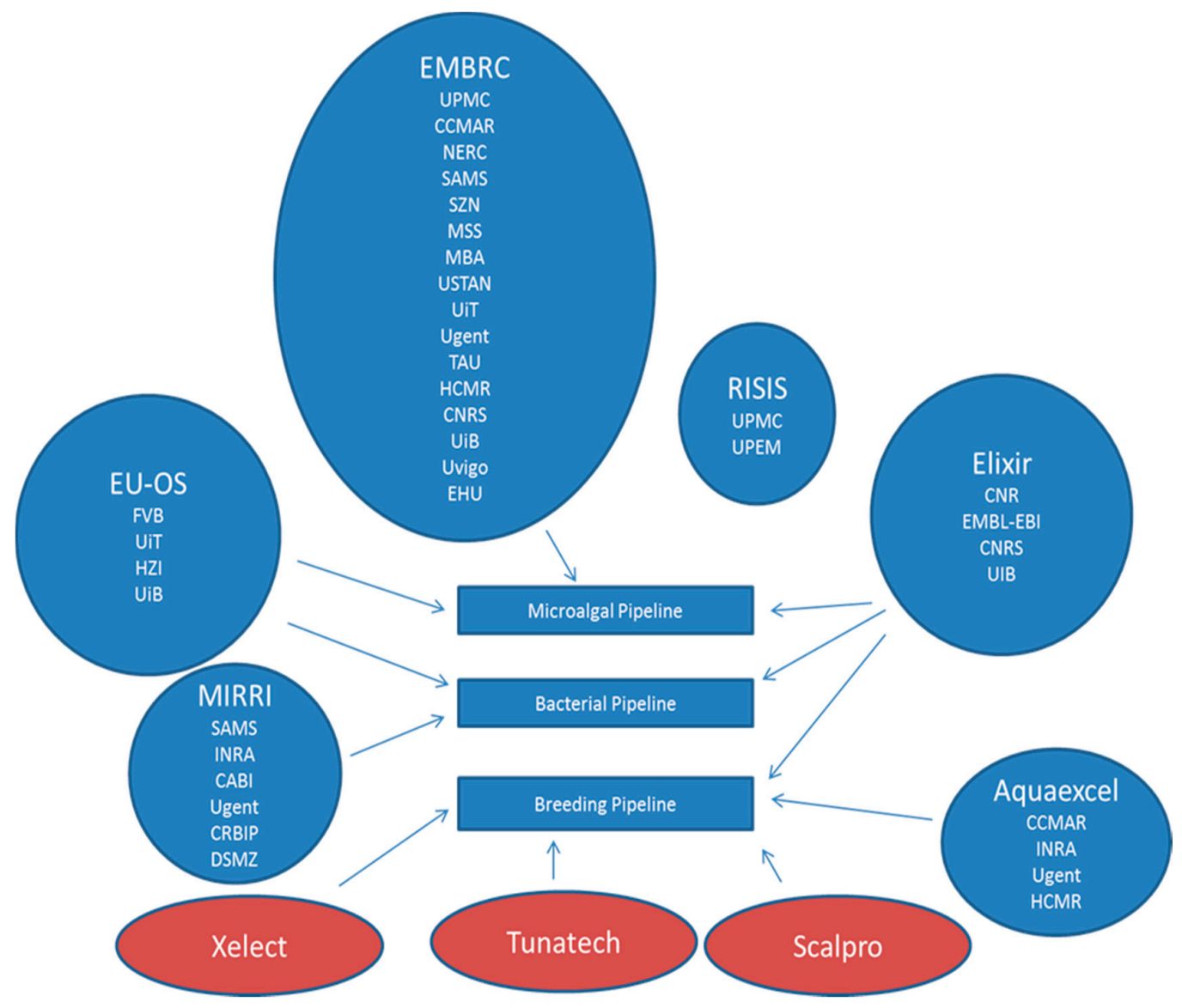

Figure 2. Research Infrastructures and industrial partners participating in the EMBRIC discovery pipelines.

\section{EMBRIC's Scientific Output So Far}

Experimental case studies have been an important activity since the very beginning of the EMBRIC project. These studies are generally meant to (i) foster collaborations amongst participating Research Infrastructures, (ii) explore how a user might utilize and combine individual components of the EMBRIC cluster, and (iii) showcase the scientific potential of EMBRIC to marine stations and industrial partners with the aim of getting them involved as collaborators at relevant stages of the respective projects. Moreover, such case studies allow thorough testing of the proposed experimental workflows, and EMBRIC case studies have thus been selected carefully to cover a broad range of the expertise present within the EMBRIC network.

\subsection{Marine Microalgal Pipeline: From Environment to Active Compounds}

Marine microalgae comprise many types of organisms adapted to different environmental conditions [1] and this translates into a great richness of potentially novel and unexplored metabolic compounds [2]. To harness this potential, one EMBRIC case study aims to facilitate discovery of natural products from unicellular algae that could be exploited for cosmetics, as well as for nutraceutical and pharmaceutical applications. To achieve this ambitious goal, knowledge on microalgal biology; skills 
in identification, isolation, and cultivation of different species; a range of bioprofiling assays; and expertise in compound isolation and characterization are needed. All these prerequisites exist within the EMBRIC network, and one of the main efforts of this case study, led by the Stazione Zoologica Anton Dohrn of Naples (SZN), is the set-up of a pipeline to connect laboratories across Europe, each contributing their expertise and resources to provide individual workflows for compound isolation, characterization, and production from microalgae (Figures 1 and 2). More specifically, this case study is divided into two main parts: (i) the discovery of new natural products and (ii) the enhancement of specific genetic traits for the efficient production of promising biomolecules by genetic engineering.

In the first part, eight partners with access to different marine ecosystems-by and large belonging to the EMBRC and MIRRI Research infrastructures-are identifying, collecting, and growing microalgal strains, thus providing the biological material for this case study. The range of laboratories involved provides access to a broad variety of algae, from polar to warm-tempered species, with different sizes, life strategies, and growth properties. Once sufficient material has been obtained, extracts are produced from algal cell pellets at University of Nice Sophia Antipolis (UNS) and subsequently shipped to SZN in duplicates for anti-proliferative and anti-oxidant assays [3]. Extracts generating hits in these bioprofiling assays are further fractionated, and obtained fractions are re-tested to identify the biomolecule responsible for the observed activity. In addition, the EU-OPENSCREEN partner HZI (Helmholtz Centre for Infection Research) also receives copies of the extracts for screening in anti-bacterial assays, for pre-metabolomics analyses that help identify the best conditions for algal cultivation, and for metabolomics profiling [4]). Metabolomics data generated in this case study will all be made available through MetaboLights [5], a publicly accessible database for metabolomics experiments and derived information. In the second part of the case study, primary assay hits will get further optimized in order to enhance desired bioactivities and maximize product yield in the respective algal strains or alternatively in heterologous expression systems. To this end, genetic engineering is being exploited to produce microalgal strains overexpressing genes linked to the production of interesting biomolecules in selected model species amenable to genetic manipulation [6-8]. Moreover, with the aim to be able to better control genetics-in a similar way to what is done for plants-experiments are conducted to induce sexual reproduction in the respective model species. More information on the results obtained can be found on the project website, where all EMBRIC deliverables are made publicly available (http:/ / www.embric.eu/deliverables).

\subsection{Marine Microbial Pipeline: From Environment to Active Compounds}

A second case study-led by $C A B$ International (CABI) — deals with releasing the potential of recalcitrant marine bacteria (Figures 1 and 2). As marine bacteria are notoriously hard to isolate and culture, the initial step of this project is to find appropriate culture conditions for those organisms in order to obtain sufficient amounts of biomass for thorough characterization and downstream experiments. To this end, substrate utilization profiles are produced at the Leibniz Institute DSMZ (German Collection of Microorganisms and Cell Cultures) using fluorescent reporters and standard molecular biology techniques to measure the response of individual bacteria to pulsed exposure to different substrates [9]. The obtained profiles then allow the subsequent design of novel cultivation media for hitherto difficult to grow bacteria. To establish and test the marine microbial pipeline five recalcitrant strains were selected and grown by the DSMZ: Jejudonia soesokkakensis, Jannaschia donghaensis, Glaciihabitans tibetensis, Sphingopyxis contaminans, and Marinifilum flexuosum. Many of these were originally isolated from biofilms in marine environments [10], thus making them good candidates for the production of interesting new natural products.

Once enough biomass has been obtained, the metabolome of the respective bacterial strains will be analysed and correlated with corresponding gene expression data generated by RNA-sequencing (RNA-seq) to gain a better understanding of the gene clusters involved in the production of the respective natural product. This information can then be used for heterologous expression experiments-performed at University of St Andrews (USTAN) - which aim to identify, clone, and express natural product gene synthesis clusters in standardized heterologous expression systems for those bacterial strains for which no suitable culture conditions can be found. 
Moreover-analogous to the first case study - example natural products from these five recalcitrant bacterial strains will be isolated and tested for interesting bioactivities by the EU-OPENSCREEN partner HZI. This part of the project also involves mode-of-action analysis for isolated and structurally characterized compounds; and interesting candidate molecules will be further optimized experimentally.

\subsection{Aquaculture Genetics and Breeding Pipeline}

More than half of all seafood consumption is now met from aquaculture following rising demand from a growing and increasingly affluent world population and stagnation of traditional capture fisheries due to over exploitation of stocks. It is estimated that by 2030, 93.6 million tons of seafood per year will be produced from aquaculture, almost twice as much as in 2008 [11]. In contrast, aquaculture production within the European Union has been static for more than a decade. Aquaculture is becoming highly industrialized, requiring innovative technological solutions to solve production bottlenecks. Major challenges include the provision of sustainably sourced feed supplies, the availability of suitable production sites, and disease and welfare issues. Part of the answer to these global challenges lies in increasing production efficiency, not least through the adoption of modern approaches to breeding and broodstock genetics to improve growth feed conversion efficiency, disease resistance, and flesh quality of farmed species [12]. EMBRIC is well placed to play an important role in supporting the uptake of modern genetics in breeding programs because of the scale of its research capabilities in 'omics' techniques (EMBRC and MIRRI), bioinformatics (ELIXIR), and aquaculture (AQUAEXCEL2020). This case study represents a pilot in the field of aquaculture genetics providing a common entry point to all three Research Infrastructures involved through partners in the United Kingdom (USTAN, Marine Science Scotland), France (French National Institute for Agricultural Research (INRA)), Greece (Hellenic Centre for Marine Research), and Norway (University of Bergen). The development of sophisticated genetic tools and the high-level skill sets in quantitative genetics and bioinformatics (Figure 1) needed to implement genetic selection is beyond the capacity of most of the companies in a highly-fragmented aquaculture sector, particularly small- and medium-sized enterprises. Innovation in aquaculture genetics is dependent on the academic sector and a relatively restricted range of specialist genetic companies. To ensure that the activities of this case study are relevant to the needs of industry, three SMEs have been included as partners (Figure 2), Scalpro A/S (a Norwegian producer of scallop and oyster spat), Tunatech $\mathrm{GmbH}$ (an innovative German aquaculture company), and Xelect Ltd. (a leading aquaculture genetics service company based in Scotland).

The layout of this case study covers all the major aspects of breeding programs including (i) trait measurements, (ii) the provision of genomic resources, and (iii) genetic marker discovery pipelines.

In the case of molluscs, established standards and ontologies for trait measurements were entirely lacking. Without such standards, it is impossible to know whether different studies have measured the same phenotypes, hampering comparative studies and precluding many types of meta-analyses (Table 2). ELIXIR and USTAN have developed data standards and metadata requirements for shellfish traits, including growth, meat yield, and toxin burden, enabling the integration of contextual information with molecular data on the European Nucleotide Archive (https:/ / www.ebi.ac.uk/ena). Feed represents around $60 \%$ of production costs and is an important target trait for genetic selection [13]. Although Feed Conversion Efficiency (FCE) shows moderate heritability, direct selection for this trait has not hitherto been possible due to technical difficulties in measuring individual feed intake over the same timescale that is required to estimate growth. The EMBRIC partner INRA from AQUAEXCEL2020 is currently developing novel approaches to measure feed conversion efficiency and solve this bottleneck in European sea bass (Dicentrarchus labrax). The feed efficiency project is in turn being used as a case study for the EMBRIC Configurator service (www.embric.eu/node/1371) being developed by ELIXIR making use of EMBRC partners as members of the expert panel to provide experimental advice. The aim is to produce a description of all elements of infrastructure used in the study such as databases, standards, formats, curation groups, analysis methods, and cloud computing capacity, and to provide advice on archiving and accessibility of the data generated. 
Table 2. List of major bottlenecks identified in the EMBRIC discovery pipelines.

\begin{tabular}{|c|c|}
\hline $\begin{array}{l}\text { Current Bottlenecks (Aquaculture Pipeline: Finfish } \\
\text { and Shellfish; Breeding Pipeline) }\end{array}$ & Suggested Solutions \\
\hline The lack of genetic tools for many species & $\begin{array}{l}\text { The use of Next Generation Sequencing techniques to develop genomic resources including draft genomes, } \\
\text { linkage maps, and marker panels }\end{array}$ \\
\hline $\begin{array}{l}\text { Poor control of inbreeding due to a lack of knowledge } \\
\text { about the pedigrees of broodstock in hatcheries }\end{array}$ & Marker-based pedigrees and pipelines for SNP panel development \\
\hline The need to identify the best broodstock & $\begin{array}{l}\text { The recording of precisely defined phenotypes involving common standards and ontologies. Methods for the } \\
\text { cost-effective measurement of genetic variation at the genomic scale. }\end{array}$ \\
\hline $\begin{array}{l}\text { Current Bottlenecks (Microalgal and Microbial } \\
\text { Pipeline: Bacteria) }\end{array}$ & Suggested Solutions \\
\hline $\begin{array}{l}\text { Cultivation of candidate organisms is often problematic, } \\
\text { with the yet uncultured and slow growing strains giving } \\
\text { low biomass production and high variability even within } \\
\text { the same species }\end{array}$ & $\begin{array}{l}\text { Research Infrastructure partners follow harmonized best practices to minimize variation and engage } \\
\text { culturomics to design improved culture conditions and coordinate efforts [14-16]. }\end{array}$ \\
\hline $\begin{array}{l}\text { Routes from crude extract assay hits to identifying and } \\
\text { purifying bioactive compounds are often problematic (for } \\
\text { example identifying the active component of extract and } \\
\text { its isolation) }\end{array}$ & $\begin{array}{l}\text { It is possible to have coordinated access across and between RIs to utilise different technologies to identify } \\
\text { and design appropriate means to get from crude extracts to pure active compounds [17] utilizing } \\
\text { infrastructure best practice [18,19]; working towards the heterologous expression of the biosynthetic gene } \\
\text { cluster of interest and/or applying a genochemetic approach (undertaken in USTAN natural product } \\
\text { discovery pipeline). }\end{array}$ \\
\hline Interoperability between data bases and data sets & Research Infrastructures agree on common standards and ontologies. \\
\hline $\begin{array}{l}\text { Lack of information to find suitable candidate bacteria } \\
\text { with relevant activities for study and how to target them } \\
\text { in the environment }\end{array}$ & $\begin{array}{l}\text { Contact MIRRI and EMBRC, who are making this knowledge available and have expertise in targeted } \\
\text { isolation [20]; keeping pace with new developments, e.g., symposia, which infrastructure partners follow } \\
\text { such as Natural Product Discovery and Development in the Genomic Era, organised by the Society for } \\
\text { Industrial Microbiology and Biotechnology: https://sim.confex.com/sim/np2018/meetingapp.cgi }\end{array}$ \\
\hline $\begin{array}{l}\text { Gaps in knowledge to be able to fully harness } \\
\text { metagenomics, proteomics, and metabolomics to identify } \\
\text { targets of interest with bioactive compounds or target } \\
\text { gene clusters in bacteria with the desired properties }\end{array}$ & $\begin{array}{l}\text { Discovery can be enhanced by gene hunting and expression in host cells [19-21]; total DNA sequence data } \\
\text { from samples can be matched with known genes and specific genes can be targeted [19]; predicting potential } \\
\text { activities from the gene clusters is now possible [22]; reliable, verifiable, and efficient monitoring of } \\
\text { biodiversity can be achieved via metabarcoding [23] }\end{array}$ \\
\hline
\end{tabular}


A fundamental requirement of aquaculture breeding programs is the need to avoid inbreeding depression, which reduces the fecundity growth and robustness of stocks. Inbreeding control requires the development of genetic marker panels and software for pedigree reconstruction and parentage assignment (Table 2). The genomic resources available for molluscs are sparse relative to finfish, which, coupled with much higher levels of nucleotide diversity and life history characteristics such as high fecundity and the incidence of hermaphroditism with potential for self-fertilization, provides particular challenges for inbreeding control [24]. USTAN and Xelect have generated genomic data sets for several cultured molluscs and are exploring the use of information-rich haplotype markers as a novel approach to parentage assignment using high throughput genotyping using sequencing methods with the aim of providing a more efficient means of inbreeding control in these species. EMBRIC partners have also used a sequence data set from the complex duplicated Atlantic salmon genome to design an efficient bioinformatics pipeline for the development and validation of parentage assignment panels, which is applicable to any aquaculture species [25]. HCMR, a partner in EMBRC, ELIXIR, and AQUAEXCEL2020, and Xelect Ltd., have been developing bioinformatics pipelines for resequencing bluefin tuna and Atlantic salmon genomes respectively. Together with collaborators at the University of Aberdeen, Xelect has mapped population variation in local genome duplications and insertions, so-called Copy Number Variation (CNVs). CNVs have the potential to become an entirely new class of selection markers in animal breeding.

\subsection{The EMBRIC Company Forums}

To further facilitate knowledge exchange between EMBRIC and the industrial sector, a first "Company Forum" was established with the aim of focusing effort on shared research priorities and disseminating the outputs of the pilot studies. EMBRIC supports open data policies and aims to encourage greater levels of business to business collaboration at precompetitive stages to the benefit of all players in the European aquaculture sector. The aquaculture Company Forum is led by TunaTech $\mathrm{GmbH}$ and supported by an advisory board drawn from academia and industry (http:/ / www.embric. eu/companyfora/aquaculture). The Company Forum held a well-attended and successful workshop at the European Aquaculture Society (EAS) Meeting in Edinburgh in September 2016 with a key note speaker from the Scottish Shellfish industry A meeting in Bergen in May 2018 discussed Advances in Industrial Aquaculture Genetics-North meets South. Further meetings of the Company Forum to disseminate the outputs of EMBRIC and encourage use of the involved Research Infrastructures by academic groups and industry are planned, for example the EAS Meeting in Montpellier in August 2018 (see http:/ / www.embric.eu/node/1191). In order to establish a similar format for the EMBRIC natural product discovery projects, a Microalgae Company Forum has been launched at the AlgaEurope conference in December 2017 in Berlin: this Forum will help academic and industrial partners with the set-up of bio-assays to identify novel bioactive compounds, and will allow access to microalgal collections and to compound libraries (http:/ / www.embric.eu/companyfora/microalgae).

\subsection{Identified Bottlenecks and Suggested Solutions}

The work conducted in the natural product discovery case studies has highlighted a number of bottlenecks to progress (see Table 2). Among the most common issues is that partners have reported inconsistency in growth when scaling up culture volumes; difficulty in obtaining sufficient biomass from some species or growth conditions; and high variability in the metabolism of different strains from the same species, which can lead to low reproducibility. Maintaining detailed records of sample growth conditions with appropriate software and databases is certainly required to minimize variation. Furthermore, while important preliminary data on bio-activities and metabolic profiles can be easily obtained for hundreds of samples, the isolation of pure compounds is currently a limiting step that cannot be easily automated; rather, it requires dedicated personnel, time, and resources. Pure compounds will certainly attract more industrial partners because of the opportunity to readily test them for different applications. 
Bottlenecks that are being addressed in the selective breeding case study include the lack trait measurement standards and availability of genomic resources for certain species, as well as the need to reduce genotyping costs in order to implement cost effective genomic selection in species with a low individual value (Table 2). Genomic selection involves genotyping and phenotyping a reference population to develop a genomic prediction algorithm to estimate breeding values that are more accurate than those derived from pedigree information alone [26]. Selection candidates are only genotyped, making the method particularly suitable for destructive traits such as disease resistance and meat yield. Genomic selection requires no knowledge of the underlying mechanism linking phenotype to genotype, and it is suitable for traits controlled by hundreds or even thousands of genes of small individual effect. In other livestock species such as dairy cattle, it relies on the use of high density SNP panels to enable brood stock to be genotyped at many tens or hundreds of thousands of loci across the genome, such that SNPs responsible for the trait variation are in linkage disequilibrium with one or more SNPs on the marker panel. A bottleneck for implementing genomic selection in aquaculture, particularly for medium sized companies, is the high cost of genotyping. Solutions to this bottleneck, in cases in which a genome is available, include genotyping the parents with a medium density SNP chip and using imputation techniques to genotype the more numerous offspring with a much lower density, allowing the use of a cheaper marker panel (Table 2).

\section{Outlook}

The examples provided above are the mere tip of the iceberg of what will be possible in the future. With the network established and the discovery pipelines proven functional, EMBRIC has now reached the point where it can start to make its resources and expertise accessible to external users from academia and industry. The EMBRIC Consortium has set aside a dedicated budget for transnational access to EMBRIC partners; the first round of external research projects is ongoing. The aim of this funding call is to start addressing major bottlenecks that have been identified so far during the setup of the discovery pipelines and that currently prevent full exploitation of the marine innovation potential. A second transnational funding call has opened in February 2018 building on the users' needs and the lessons learned from the first round of funding.

Generally, transnational access is offered by 15 access providers belonging to four different Research Infrastructures (AQUAEXCEL2020, EMBRC, EU-OPENSCREEN, and MIRRI) in seven European countries. Users of the transnational access program can be scientists from academia or industry, with their home institutions based in EU member states or associated countries (as defined by the Horizon 2020 programme). Access for user groups with a majority of users not working in EU Member States or Associated Countries is limited to $20 \%$ of the total amount of units of access provided.

Because of its multidisciplinary nature, the second round of transnational access funding will continue to raise awareness of marine translational science across many different research areas and will thus help to accelerate blue innovation through cross-disciplinary collaboration-as the Japanese poet Ryunosuke Satoro once put it: "Individually, we are one drop. Together, we are an ocean".

Funding: This research was funded by European Union's Horizon 2020 research and innovation programme under grant agreement No 654008.

Acknowledgments: The manuscript has benefited from the input of the whole project consortium (see http: / / www.embric.eu/partner-list/all) and, in particular, Jörg Overmann and Cendrella Lepleux (DSMZ), Mark Brönstrup and Michael Hensler (HZI), Bernard Kloareg (SB-Roscoff), Mery Pina (UPMC) Francois-Yves Bouget (OOB), Adrianna Ianora (SZN), Mohamed Mehiri (UNS), Angela Falciatore (UPMC), and Philip Gribbon (IME Screening Port). The results, discussion, and opinions reflect the authors' view, and the European Commission is not responsible for any use that may be made of the information it contains.

Conflicts of Interest: The authors declare no conflict of interest. 


\section{Abbreviations}

\begin{tabular}{|c|c|}
\hline EMBRIC & European Marine Biological Research Infrastructure Cluster \\
\hline EMBRC & European Marine Biological Resource Center \\
\hline Elixir & A distributed infrastructure for life-science information \\
\hline EU-OS & EU-Openscreen \\
\hline ESFRI & European Strategy Forum on Research Infrastructures \\
\hline SMEs & Small and Medium-sized Enterprises \\
\hline MIRRI & Microbial Resources Research Infrastructure \\
\hline ECBL & European Chemical Biology Library \\
\hline RISIS & Research Infrastructure for Research and Innovation Policy Studies \\
\hline IP & Intellectual property \\
\hline NPs & Natural products \\
\hline RNA-seq & RNA-sequencing \\
\hline CABI & CAB International \\
\hline CCMAR & Centro de Ciencias do Mar do Algarve \\
\hline CNR & Consiglio Nazionale delle Ricerche \\
\hline CNRS & Centre national de la recherche scientifique \\
\hline CRBIP & Biological Resource Center Institut Pasteur \\
\hline DSMZ & Deutsche Sammlung von Mikroorganismen und Zellkulturen GMBH \\
\hline EHU & European Humanities University, University of the Basque \\
\hline EMBL-EBI & European Molecular Biology Laboratory, European Bioinformatics Institute \\
\hline FVB & Forschungsverbund Berlin E.V. \\
\hline HCMAR & Hellenic Centre for Marine Research \\
\hline HZI & Helmholtz-Zentrum fuer Infektionsforschung GMBH \\
\hline IME & Fraunhofer ScreeningPort IME \\
\hline INRA & Institut National de la Recherche Agronomique \\
\hline MBA & Marine Biological Association of the United Kingdom \\
\hline MDA & Multiple displacement amplification \\
\hline MSS & Marine Scotland Science \\
\hline NERC & National Environment Research Council \\
\hline OOB & Observatoire Océanologique of Banyuls \\
\hline SAMS & Scottish Association for Marine Science \\
\hline $\mathrm{SZN}$ & Stazione Zoologica Anton Dohrn of Naples \\
\hline TAU & Tel Aviv University \\
\hline UNS & Université de Nice Sophia Antipolis \\
\hline UPMC & University Pierre and Marie Curie \\
\hline UPEM & Université Paris Est Marne La Vallée \\
\hline UiB & University of Bergen \\
\hline UiT & The Arctic University of Norway \\
\hline UGent & University of Gent \\
\hline USTAN & University of St Andrews \\
\hline Uvigo & University of Vigo \\
\hline
\end{tabular}

\section{References}

1. Parker, M.S.; Mock, T.; Armbrust, E.V. Genomic Insights into Marine Microalgae. Annu. Rev. Genet. 2008, 42, 619-645. [CrossRef] [PubMed]

2. Maeda, Y.; Yoshino, T.; Matsunaga, T.; Matsumoto, M.; Tanaka, T. Marine microalgae for production of biofuels and chemicals. Curr. Opin. Biotechnol. 2017, 50, 111-120. [CrossRef] [PubMed]

3. Sansone, C.; Galasso, C.; Orefice, I.; Nuzzo, G.; Luongo, E.; Cutignano, A.; Romano, G.; Brunet, C.; Fontana, A.; Esposito, F.; et al. The green microalga Tetraselmis suecica reduces oxidative stress and induces repairing mechanisms in human cells. Sci. Rep. 2017, 7, 41215. [CrossRef] [PubMed]

4. Depke, T.; Franke, R.; Bronstrup, M. Clustering of MS2 spectra using unsupervised methods to aid the identification of secondary metabolites from Pseudomonas aeruginosa. J. Chromatogr. B 2017, 1071, 19-28. [CrossRef] [PubMed]

5. Haug, K.; Salek, R.M.; Conesa, P.; Hastings, J.; de Matos, P.; Rijnbeek, M.; Mahendraker, T.; Williams, M.; Neumann, S.; Rocca-Serra, P.; et al. Metabolights-An open-access general-purpose repository for metabolomics studies and associated meta-data. Nucleic Acids Res. 2013, 41, D781-D786. [CrossRef] [PubMed]

6. Falciatore, A.; Casotti, R.; Leblanc, C.; Abrescia, C.; Bowler, C. Transformation of nonselectable reporter genes in marine diatoms. Mar. Biotechnol. 1999, 1, 239-251. [CrossRef] [PubMed] 
7. Van Ooijen, G.; Knox, K.; Kis, K.; Bouget, F.Y.; Millar, A.J. Genomic Transformation of the Picoeukaryote Ostreococcus tauri. J. Vis. Exp. 2012, e4074. [CrossRef] [PubMed]

8. Sabatino, V.; Russo, M.T.; Patil, S.; d'Ippolito, G.; Fontana, A.; Ferrante, M.I. Establishment of Genetic Transformation in the Sexually Reproducing Diatoms Pseudo-nitzschia multistriata and Pseudo-nitzschia arenysensis and Inheritance of the Transgene. Mar. Biotechnol. 2015, 17, 452-462. [CrossRef] [PubMed]

9. Bruns, A.; Hoffelner, H.; Overmann, J. A novel approach for high throughput cultivation assays and the isolation of planktonic bacteria. FEMS Microbiol. Ecol. 2003, 45, 161-171. [CrossRef]

10. Gich, F.; Janys, M.A.; Konig, M.; Overmann, J. Enrichment of previously uncultured bacteria from natural complex communities by adhesion to solid surfaces. Environ. Microbiol. 2012, 14, 2984-2997. [CrossRef] [PubMed]

11. World Bank. Fish to 2030: Prospects for Fisheries and Aquaculture (English); Agriculture and Environmental Services Discussion Paper No. 3; World Bank: Washington, DC, USA, 2013.

12. Gjedrem, T.; Robinson, N. Advances by Selective Breeding for Aquatic Species: A Review. Agric. Sci. 2014, 5, 1152-1158. [CrossRef]

13. Daule, S.; Vandeputte, M.; Vergnet, A.; Guinand, B.; Grima, L.; Chatain, B. Effect of selection for fasting tolerance on feed intake, growth and feed efficiency in the European sea bass Dicentrarchus labrax. Aquaculture 2014, 420, S42-S49. [CrossRef]

14. Nichols, D.; Cahoon, N.; Trakhtenberg, E.M.; Pham, L.; Metha, A.; Belanger, A.; Kanigan, T.; Lewis, K.; Epstein, S.S. Use of Ichip for High-Throughput In Situ Cultivation of “Uncultivable” Microbial Species. Appl. Environ. Microbiol. 2010, 76, 2445-2450. [CrossRef] [PubMed]

15. Lagier, J.C.; Armougom, F.; Million, M.; Hugon, P.; Pagnier, I.; Robert, C.; Bittar, F.; Fournous, G.; Gimenez, G.; Maraninchi, M.; et al. Microbial culturomics: Paradigm shift in the human gut microbiome Study. Clin. Microbiol. Infect. 2012, 18, 1185-1193. [CrossRef] [PubMed]

16. Cheng, C.; Sun, J.; Zheng, F.; Wu, K.; Rui, Y. Molecular identification of clinical “difficult-to-identify” microbes from sequencing $16 \mathrm{~S}$ ribosomal DNA and internal transcribed spacer 2. Ann. Clin. Microbiol. Antimicrob. 2014, 13, 1. [CrossRef] [PubMed]

17. Strobel, G.; Daisy, B. Bioprospecting for microbial endophytes and their natural products. Microbiol. Mol. Biol. Rev. 2003, 67, 491-502. [CrossRef] [PubMed]

18. Bucar, F.; Wubea, A.; Schmidb, M. Natural product isolation-How to get from biological material to pure compounds. Nat. Prod. Rep. 2013, 30, 525-545. [CrossRef] [PubMed]

19. Trindade, M.; van Zyl, L.; Navarro-Fernández, J.; Abd Elrazak, A. Targeted Metagenomics as a Tool to Tap Into Marine Natural Product Diversity for the Discovery and Production of Drug Candidates. Front. Microbiol. 2015. [CrossRef] [PubMed]

20. Milshteyn, A.; Schneider, J.S.; Brady, S.F. Mining the Metabiome: Identifying Novel Natural Products from Microbial Communities. Chem. Biol. 2014, 21, 1211-1223. [CrossRef] [PubMed]

21. Harvey, A.L. Natural products in drug discovery. Drug Discov. Today 2008, 13, 894-901. [CrossRef] [PubMed]

22. Dejong, C.A.; Chen, G.M.; Li, H.; Johnston, C.W.; Edwards, M.R.; Rees, P.N.; Skinnider, M.A.; Webster, A.L.H.; Magarvey, N.A. Polyketide and nonribosomal peptide retro-biosynthesis and global gene cluster matching. Nat. Chem. Biol. 2016, 12, 1007-1014. [CrossRef] [PubMed]

23. Ji, Y.; Ashton, L.; Pedley, S.M.; Edwards, D.P.; Tang, Y.; Nakamura, A.; Kitching, R.; Dolman, P.M.; Woodcock, P.; Edwards, F.A.; et al. Reliable, verifiable and efficient monitoring of biodiversity via metabarcoding. Ecol. Lett. 2013, 16, 1245-1257. [CrossRef] [PubMed]

24. Astorga, M.P. Genetic considerations for mollusk production in aquaculture: Current state of knowledge. Front. Genet. 2014, 5, 435. [CrossRef] [PubMed]

25. Holman, L.E.; de la Serrana, D.G.; Onoufriou, A.; Hillestad, B.; Johnston, I.A. A workflow used to design low density SNP panels for parentage assignment and traceability in aquaculture species and its validation in Atlantic salmon. Aquaculture 2017, 476, 59-64. [CrossRef]

26. Meuwissen, T.; Hayes, B.; Goddard, M. Accelerating improvement of livestock with genomic selection. Annu. Rev. Anim. Biosci. 2013, 1, 221-237. [CrossRef] [PubMed]

(C) 2018 by the authors. Licensee MDPI, Basel, Switzerland. This article is an open access article distributed under the terms and conditions of the Creative Commons Attribution (CC BY) license (http://creativecommons.org/licenses/by/4.0/). 